\title{
Intracluster Planetary Nebulae in the Hydra I Cluster ${ }^{\star}$
}

\author{
G. Ventimiglia ${ }^{1,2, \star \star}$ M. Arnaboldi ${ }^{1}$ and O. Gerhard ${ }^{2}$ \\ 1 ESO, Karl-Schwarzschild-Strasse 2, D-85748 Garching bei Muenchen \\ ${ }^{2}$ Max Planck Institute fuer Extraterrestrische Physics, Giesenbachstrasse, D-85741 Garching bei Muenchen
}

Received 2008 Sep 18, accepted 2008 Sep 22

Published online 2008 Nov 20

Key words galaxy clusters: general - galaxy clusters: specific (Hydra I) - Planetary Nebulae

Using the Multislit Imaging Spectroscopy (MSIS) technique at the FORS2 spectrograph on VLT-UT1, we have identified 60 Intracluster Planetary Nebula (ICPN) candidates associated with the Intracluster Light (ICL) in the central region of the Hydra I cluster. Hydra I is a medium compact, relatively near $(\sim 50 \mathrm{Mpc})$, rich cluster in the southern hemisphere. Here we describe the criteria used to select emission sources and present the evidence for these PN candidates to be associated with the ICL in the Hydra I cluster. We also show, using the luminosity-specific planetary nebulae number, the $\alpha$ parameter, that the expected number of PNs associated with the stellar population of the central cD galaxy NGC 3311 in the cluster is close to the number of PNs detected.

(C) 2008 WILEY-VCH Verlag GmbH \& Co. KGaA, Weinheim

\section{Introduction}

Diffuse intracluster light (ICL) has been observed both in nearby and intermediate redshift clusters (Mihos et al. 2005, Feldmeier et al. 2004). Observations show that the diffuse light in galaxy clusters amounts to $\sim 10 \%$ of the total light emitted by the cluster galaxies (Aguerri et al. 2005; Zibetti et al. 2005).

Cosmological simulations of structure formation predict intracluster stars to be lost from galaxies in interactions during the assembly of galaxy clusters (Napolitano et al. 2003; Murante et al. 2004; Willman et al. 2004; Sommer-Larsen, Romeo \& Portinari 2005). Simulations suggest that most of the diffuse light in galaxy cluster cores originates from mergers that lead to the formation of the brightest cluster galaxy and of the other massive galaxies, while the tidal stripping mechanism dominates the formation of the ICL at larger radii (Murante et al. 2007).

The study of the properties of this diffuse component has then an important role in understanding the mechanisms relevant for the evolution of galaxies in high density environments, and the formation history, dynamics and merging status of clusters.

Wide field imaging has already shown the morphological complexity of the ICL: studies of the Virgo and Coma cluster ICL have shown that it is characterized by tidal features like streamers and extended galaxy halos (Adami et al. 2005; Mihos et al. 2005). Due to its intrinsically low surface brightness, $\mu_{V}>28.5 \mathrm{mag} \operatorname{arcsec}^{-2}$ (Feldmeier

\footnotetext{
* Based on data collected at ESO, Chile (ESO program 076.B-0641(B))

$\star \star$ Corresponding author: e-mail: gventimi@eso.org
}

et al. 2004; Mihos et al. 2005), the kinematics of ICL can only be studied using the Intracluster Planetary Nebulae (ICPNs) associated with this stellar component (Arnaboldi et al. 2004, Gerhard et al. 2007, Doherty et al. 2008). ICPNs are relatively easy to detect because their spectra are characterized by two strong emission lines: [OIII] $\lambda 4959 \AA$ and $[\mathrm{OIII}] \lambda 5007 \AA$, with relative flux ratio $1: 3$.

The goal of our project is to measure the velocity distribution of the PNs associated with the diffuse light in the central region of the nearby Hydra I cluster (Abell 1060, $D \sim 50 \mathrm{Mpc}, z \sim 0.0126$ ), a medium compact, non-cooling flow, rich cluster in the southern hemisphere. This will be presented in a forthcoming paper. Here we present the selection criteria and the evidence for the detection of PNs associated with the ICL in Hydra I.

At the distance of the Hydra I cluster, the flux of the O[III] $\lambda 5007 \AA$ emission line of a PN is less than $8 \times 10^{-18}$ $\mathrm{erg} \mathrm{s}^{-1} \mathrm{~cm}^{-2}$, therefore we need to reduce substantially the noise from the night sky in order to detect these lines. This is possible by using the Multislit Imaging Spectroscopy technique (MSIS: Gerhard et al. 2005, Arnaboldi et al. 2007). In what follows we define $m_{5007}=-2.5 \log F_{5007}-13.74$ (the Jacoby formula, Jacoby et al. 1989), where $F_{5007}$ is the integrated flux in the [OIII] $\lambda 5007 \AA$ emission line, and we assume a distance of $50 \mathrm{Mpc}$ for the Hydra I cluster implying $1 " \sim 0.24 \mathrm{Kpc}$.

In the next section we present the MSIS observations carried out with FORS2 on UT1. In Section 3 we summarize the data reduction. We present the adopted selection criteria to identify the emission sources and the evidence for PNs associated with the ICL in the Hydra I cluster in Section 4. In Section 5 we show that the number density of PN candi- 
dates detected is consistent with that expected for the stellar population associated with the central $\mathrm{cD}$ galaxy of the cluster, NGC 3311. In Section 6 we summarize our results.

\section{Observations}

\subsection{The Multislit Imaging Spectroscopy technique}

The MSIS technique consists of the combined use of a mask of parallel slits, a narrowband filter centered around the redshifted [OIII] $\lambda 5007 \AA$ line, and a dispersing element. It is a blind search technique and allows one to obtain spectra of all PNs (and other emission line objects) that happen to lie behind the mask slits. Because the [OIII] emission lines from PNs are only $\sim 30 \mathrm{~km} / \mathrm{s}$ wide (Arnaboldi et al. 2008), when dispersed at spectral resolution $R=\lambda / \Delta \lambda \sim 6000$, their entire flux falls into a small number of pixels in the two-dimensional spectrum, determined by the slit width and seeing. By dispersing the sky noise on many pixels, the technique allows measurement of very faint fluxes. We can detect PNs with magnitudes $\sim 1.2$ mag below the bright cutoff of the Planetary Nebulae Luminosity Function (PNLF; Ciardullo et al. 1998) and their positions and radial velocities can be measured at the same time.

\subsection{Observational set up}

Data were collected in visitor mode during 2006 March 2628, using the FORS2 spectrograph on UT1. The observed area covers the central region of the Hydra I cluster, around NGC 3311, at $\alpha=10 \mathrm{~h} 36 \mathrm{~m} 42.8 \mathrm{~s}, \delta=-27 \mathrm{~d} 31 \mathrm{~m} 42 \mathrm{~s}$ (J2000).

The FORS2 field of view (FOV) is $6^{\prime} .8 \times 6^{\prime} .8$ wide, corresponding to $\sim 10000 \mathrm{kpc}^{2}$, and is imaged onto a mosaic of two CCDs, rebinned $2 \times 2$ in the readout. We used two narrow band filters, one centered at $\lambda=5045 \AA$ and a second one at $\lambda=5095 \AA$, both with a FWHM of $60 \AA$. We cover, in this way, the whole range of cluster line-ofsight (LOS) velocities and, for fast, $v_{\mathrm{LOS}} \geq 4000 \mathrm{~km} \mathrm{~s}^{-1}$, and bright PNs, $m_{5007} \leq 29.3$, the [OIII] $\lambda 5007 \AA$ emission line falls in the red filter and the [OIII] $\lambda 4959 \AA$ line in the blue filter. Spectra were obtained with the GRIS-600B grism, which has a spectral resolution of $0.75 \AA /$ pixel at $5075 \AA$. The MSIS mask is made of $24 \times 21$ slits, each of them 0 ". .8 wide and 17 ". 5 long. Each slit is projected along the dispersion axis onto $\sim 40$ rebinned pixels. The effective area imaged by the slits is $\sim 7056 \operatorname{arcsec}^{2}$, that is $\sim 4.5 \%$ of the whole FORS2 FOV. In order to cover the whole field the MSIS mask was stepped 15 times on the sky to cover the region between two adjacent slits. For each mask position 3 exposures, of $1200 \mathrm{sec}$ each, were taken, ensuring a proper cosmic ray subtraction. The seeing during the three observing nights was in the range from 0 ". 6 to 1 ".5.

Arclamp calibration frames were acquired for the wavelength calibration, as well as flats and bias images. The flux calibration was done using long slit observations of the standard star LTT7379 with narrow band filter plus Grism.
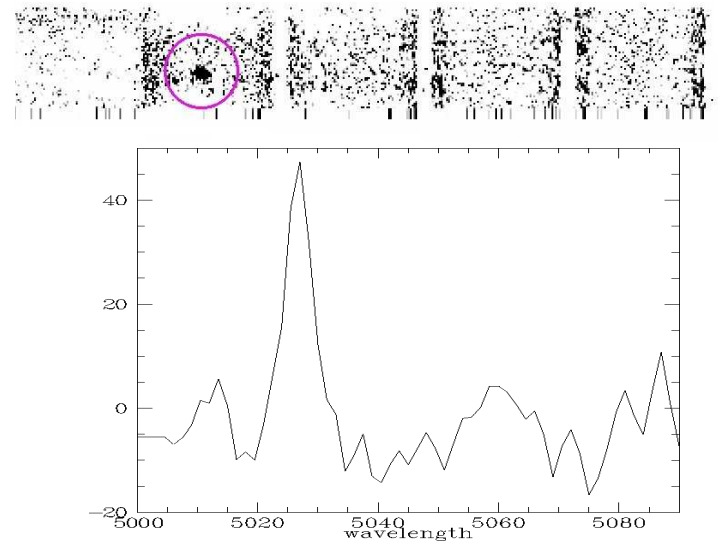

Fig. 1 Upper panel: 2-D spectrum of a PN candidate. The dispersion axis is along the vertical direction. The emission line falls onto about 6 pixels both in the spatial and spectral direction. Lower panel: 1-D spectrum of the same PN candidate. The emission line has the same FWHM as the arc lamp lines, showing that the observed emission line is unresolved in wavelength.

\section{Data reduction}

Data reduction was carried out following the procedure described in Arnaboldi et al. (2007). After bias subtraction, the images were properly co-added and the continuum light from the two main galaxies was subtracted, with an fmedian filtering using a window of $19 \times 35$ pixels.

Then we extracted and rectified the 2D spectra (Fig. 1) of the emission sources and performed the wavelength and flux calibration. The total number of emission sources detected is 95 . On the basis of the flux calibration the $1 \sigma$ limit on the continuum is $\sim 7 \times 10^{-20} \mathrm{erg} \mathrm{cm}^{-2} \mathrm{~s}^{-1} \AA^{-1}$.

A first classification of the emission line objects can be carried out according to the following criteria:

- PN candidates: unresolved emission line objects, both in space and in wavelength, with no continuum;

- background objects candidates: continuum sources with unresolved or resolved emission line.

Of the 95 emission lines sources identified, 60 were classified as possible PN candidates, the remaining sources as background object candidates. Monochromatic point like emissions appear in the final images as unresolved sources with a width of $\sim 6$ pixels both in the spatial and in the wavelength direction. The final spectral resolution is $\sim 4.5 \AA$ or $140 \mathrm{~km} \mathrm{~s}^{-1}$. Magnitudes for the PN candidates were computed using the Jacoby formula.

\section{Selection Criteria for the $P N$ population in the Hydra cluster: the wavelength-magnitude plane}

We now describe the physical properties of PNs belonging to the Hydra I ICL in a two dimensional wavelengthmagnitude space, as shown in Fig2 2 




Fig. 2 The plot shows the wavelength vs magnitude space in which we describe the physical properties of a PN population at $50 \mathrm{Mpc}$ and bound to the Hydra I cluster. Blue and red crosses are the PN candidates detected in the MSIS images. The various lines and the characteristics of the 4 regions identified in the plot are explained in the text.

A PN population is characterized by a bright cut-off of the PNLF, which according to Ciardullo et al. (1998) has absolute magnitude of the [OIII] $\lambda 5007 \AA$ emission line of $M^{*}=-4.48$. At the Hydra I cluster distance ( $m-$ $M=33.49$ ), this corresponds to an apparent magnitude of $m_{5007}=29.1$ (plotted as the dotted horizontal line in Fig 2]. The apparent magnitude for the [OIII] $\lambda 4959 \AA$ emission line is 1.2 mag fainter.

The red inclined line in Fig 2 shows the apparent magnitude of a PN at the PNLF bright cutoff, as a function of the Hubble flow distance, and the blue line shows the same dependence for the [OIII] $\lambda 4959 \AA$ emission line. If the lineof-sight velocity corresponded only to the Hubble flow, then the magnitude of the two emission lines, [OIII] $\lambda 4959 \AA$ and [OIII] $\lambda 5007 \AA$ of a PN fainter than the bright cut-off would fall on the left side of the blue and red line, respectively.

The systemic velocity of the Hydra I cluster $v_{\text {Hydra }}=$ $3683 \mathrm{~km} \mathrm{~s}^{-1}$ and its velocity dispersion $\sigma_{\text {Hydra I }}=724 \mathrm{~km}$ $\mathrm{s}^{-1}$ (Christlein \& Zabludoff 2003) identify a wavelength range in which [OIII] emissions from objects orbiting in the Hydra I cluster potential can be observed. At the systemic velocity of the cluster, the [OIII] emission lines are redshifted to $\lambda=5068 \AA$ and $\lambda=5020 \AA$, respectively. In Fig 2 the central green line is at $\lambda=5068 \AA$ where the [OIII] $\lambda 5007 \AA$ line is redshifted to the cluster systemic velocity. The other two vertical green lines are at the wavelengths bounding the $\pm 3 \sigma$ velocity range, respectively at $\lambda=5032 \AA$ and $\lambda=5104 \AA$.

The black continuous lines in Fig 2 show the measured profiles of the narrow band filters, $T(\lambda)$, normalized so that the maximum transmission is at the PNLF bright cutoff.
They are centered respectively at $\lambda=5045 \AA$ and $\lambda=$ $5095 \AA$ and have a FWHM of $\sim 60 \AA$.

Considering the magnitude limit and wavelength range specified, we can identify different areas in this wavelengthmagnitude space, separated by black horizontal lines:

- Region \#1 According to their velocity and magnitude all emission lines are [OIII] $\lambda 5007 \AA$.

- Region \#2 It is the region where, considering the flux and wavelength, we can find both faint [OIII] $\lambda 5007 \AA$ or [OIII] $\lambda 4959 \AA$ emission lines.

- Region \#3 Here we have high flux and red wavelengths so that we can see the bright [OIII] $\lambda 5007 \AA$ emissions. For such emissions we expect to detect the corresponding [OIII] $\lambda 4959 \AA$ in region \#2.

- Region \#4 Here the emissions are most likely [OIII] $\lambda 5007 \AA \AA$. In principle, we may find both [OIII] $\lambda 4959 \AA$ and $[\mathrm{OIII}] \lambda 5007 \AA$; however, if an emission were identified as [OIII] $\lambda 4959 \AA$ its LOS velocity would be about $8600 \mathrm{~km} \mathrm{~s}^{-1}$ which is more than $6 \sigma$ away from the cluster systemic velocity. This PN would then not be bound to Hydra I and its velocity driven by the Hubble flow. Then its [OIII] $\lambda 4959 \AA$ magnitude should fall on the left side of the blue line. Therefore [OIII] $\lambda 4959 \AA$ emission lines in this region are ruled out.

We can now populate the wavelength-magnitude plane with the PN candidates detected in the MSIS spectra. This gives us important information to validate the PNs catalogue. In the plot the blue and red crosses are the PN candidates detected in the wavelength ranges covered by the blue and red filters respectively.

The first result is that the fluxes of these candidates are all consistent with those of PNs at the distance of the Hydra I cluster, in the range from $1.7 \times 10^{-18}$ to $8.4 \times 10^{-18} \mathrm{erg}$ $\mathrm{s}^{-1} \mathrm{~cm}^{-2}$, i.e. $30.7>m_{5007}>28.9$.

The second is that for the two bright [OIII] $\lambda 5007 \AA$ emission sources identified in region \#3, the corresponding [OIII] $\lambda 4959 \AA$ have been identified in region $\# 2$ : these objects are encircled in magenta. All others emission sources in the plot are [OIII] $\lambda 5007 \AA$ if in the cluster.

Moreover, we know that the $1 \sigma$ continuum upper limit flux in both filters is $\sim 7 \times 10^{-20} \mathrm{erg} \mathrm{cm}^{-2} \mathrm{~s}^{-1} \AA^{-1}$. Considering that the compact HII regions detected in the Virgo cluster (Gerhard et al. 2002) have a V-band continuum flux of $\sim 8.2 \times 10^{-19} \mathrm{erg} \mathrm{cm}^{-2} \mathrm{~s}^{-1} \AA^{-1}$, corresponding to $\sim$ $9.6 \times 10^{-20} \mathrm{erg} \mathrm{cm}^{-2} \mathrm{~s}^{-1} \AA^{-1}$ at the distance of the Hydra I cluster, this allows us to rule out from our sample compact HII regions such as or brighter than those observed in Virgo. Also, from the continuum upper limit flux we calculate that the Equivalent Width (EW) of the most luminous candidates is $E W>90 \AA$. This value is larger than those of [OII] emitting background galaxies at $z=0.37$, which have $E W_{[O I I]}<50$ (Hogg et al. 1998). Therefore, we can rule out all contaminations to our sample except a few background Ly $\alpha$ galaxies.

In summary, from analyzing the PN candidates in the wavelength-magnitude plane we learn that: 
- fluxes are consistent with PNs at the distance of the Hydra I cluster; the only possible contaminants are Ly $\alpha$ emitters at high redshift;

- objects in magenta circles can reliably be classified as PNs because we are able to see both their [OIII] $\lambda 5007 \AA$ and [OIII] $\lambda 4959 \AA$ emission lines;

- for all other PNs we have detected the [OIII] $\lambda 5007 \AA$ at the LOS velocity expected for objects bound to the Hydra I cluster.

\section{The PN population associated with the stellar population of the Hydra I cluster}

We now evaluate the expected number of PNs associated with the total stellar luminosity of NGC 3311 and we test whether the number of detected candidates is consistent with that expected given the sampled light, the flux limit and the average color of the stellar population. The luminosityspecific planetary nebulae number, the so called $\alpha$ parameter, specifies the number of PNs associated with the amount of light emitted by the stellar population of a galaxy (Jacoby et al. 1980). According to Buzzoni, Arnaboldi, \& Corradi (2006) this is defined as $\alpha=N_{P N} / L_{B}$, where $N_{P N}$ is the number of PNs of a galaxy and $L_{B}$ is its total stellar luminosity in the B-band. As shown in Buzzoni et al. (2006), the $\alpha$ parameter is correlated with the $(B-V)$ colour index of the galaxies.

To compare the theoretical number of PNs expected for NGC 3311 in Hydra I with the number detected in the MSIS images we have gone through the following steps. We took $(B-V)=1.04$ for NGC 3311 from Prugniel \& Heraudeau (1998) and the colour excess $E(B-V)=0.079$ from the NED database to determine the $(B-V)_{0}=0.961$ colour corrected for extinction. From this value we obtained $\alpha=$ $1.5 \times 10^{-7}$ using Table \#6 in Buzzoni et al. (2006). To calculate the predicted number of PNs in the MSIS images a completeness correction is necessary. Since with our technique we detect only PNs down to 1.2 magnitude from the PNLF bright cut-off, the number we need is $\alpha_{1.2}$. This is simply a fraction of the total luminosity-specific PN number: $\alpha_{1.2}=$ $\alpha / 30=5 \times 10^{-9}$ (Buzzoni et al. 2006). The total B-band luminosity of the diffuse light from NGC 3311's envelope in the FORS2 field, excluding the central 34" where the galaxy is brighter than the sky, is $L_{B}=6.8 \times 10^{10} L_{\odot}\left(M_{B}=\right.$ -21.7 from Vaesterberg, Lindblad \& Jorsater 1991). Then the total number of PNs expected in the FOV is: $N_{P N}=$ $\alpha_{1.2} / L_{B} \sim 114$. Because each MSIS mask covers an area of about $4.5 \%$ of the total FORS2 area, we thus expect to see $114 \times 0.045 \sim 5$ PNs per mask, i.e. $\sim 75$ PNs in the total MSIS image sample. This is consistent within $1.5 \sigma$ with the actual number of PN candidates, 60 .

\section{Conclusions}

In this work, we have presented MSIS observations of PN candidates associated with the diffuse light around the cen- tral cD galaxy NGC 3311 in the Hydra I cluster. We have discussed criteria used for selecting these emission sources and have analyzed their properties in the wavelength-magnitude plane. In total, we have identified $60 \mathrm{PN}$ candidates around NGC 3311, consistent with the number of PNs expected from the diffuse envelope of this galaxy.

In the next steps of our analysis we will focus on the kinematics of the diffuse stellar population in the halo of NGC 3311. We will study the histogram of the LOS velocity distribution of the PNs candidates to obtain information about possible substructures in the ICL and the merging status of the cluster. Together with the X-ray temperature profile (Yamasaki et al. 2002) and the surface brightness profiles of the stellar light this will give us constraints on the orbit distribution in the halo of this $\mathrm{cD}$ galaxy.

Acknowledgements. G. Ventimiglia is supported by an ESO studentship.

\section{References}

Adami, C. et al.: 2005a, A\&A 429,39

Aguerri, J.A.J. et al.: 2005, ApJ 129, 2585

Arnaboldi, M. et al.: 2004, ApJ 614, L33

Arnaboldi, M. et al.: 2007, PASJ 59, 419

Arnaboldi, M. et al.: 2008, ApJ 674, 17

Buzzoni, A., Arnaboldi, M. \& Corradi, R.L.M.: 2006, MNRAS 368,877

Christlein, D. \& Zabludoff, A.I.: 2003, ApJ 591, 764

Ciardullo, R., Jacoby, G.H., Feldmeier, J.J., \& Bartlett, R.E.: 1998, ApJ 492, 62

Doherty, M. et al.: 2008, submitted to A\&A

Feldmeier, J.J., Ciardullo, R., Jacoby G.H., \& Durell, P.R.: 2004, ApJ 615,196

Gerhard, O., Arnaboldi, M., Freeman, K.C., Okamura, S.: 2002, ApJ 580, L121

Gerhard, O. et al.: 2005, ApJ 621, L93

Gerhard, O. et al.: 2007, A\&A 468, 815

Hogg, D.W., Cohen, J.G., Blandford, R., Pahre, M.: 1998, ApJ 504,622

Jacoby, G.H.: 1980, ApJS 42, 1

Jacoby, G.H.: 1989, ApJ 339, 39

Mihos, J.C.,Harding, P., Feldmeier, J., Morrison, H.: 2005, ApJ 631, L41

Murante, G. et al.: 2004, ApJ 607, L83

Murante, G. et al.: 2007, MNRAS 377, 2

Napolitano, R. et al.: 2003, ApJ 594, 172

Prugniel, Ph. \& Heraudeau, Ph.: 1998, A\&A 128, 299

Sommer-Larsen, J., Romeo, A. D., Portinari, L.: 2005, MNRAS 357,478

Vaesterberg, A.R., Lindblad, P.O. \& Jorsater, S.: 1991, A\&A 247, 335

Willman, B., Governato, F., Wadsley, J., Quinn, T.: 2004, MNRAS 355,159

Yamasaki, N.Y. et al.: 2002, ApJ 578, 833

Zibetti, S., White, S.D.M., Schneider, D.P., Brinkmann, J.: 2005, MNRAS 358, 949 